\title{
Identification of the autotransporter Pet toxin in Proteus mirabilis strain isolated from patients with urinary tract infections
}

\author{
Luis Raúl Gutiérrez-Lucas ${ }^{1}$, Guillermo Mendoza-Hernández ${ }^{2}$, Bertha González-Pedrajo ${ }^{3}$, \\ Carlos Eslava-Campos ${ }^{2}$, Jaime Bustos-Martínez ${ }^{4}$, Teresita Sainz-Espuñes ${ }^{{ }^{*}}$ \\ ${ }^{1}$ Departamento de Sistemas Biológicos, Universidad Autónoma Metropolitana-Xochimilco, México City, México \\ ${ }^{2}$ Departamento de Bioquímica, Facultad de Medicina, UNAM, México City, México \\ ${ }^{3}$ Instituto de Fisiología Celular, UNAM, México City, México \\ ${ }^{4}$ Departamento de Atención a la Salud, Universidad Autónoma Metropolitana-Xochimilco, México City, México \\ Email: ${ }^{\text {trsainz@correo.xoc.uam.mx }}$
}

Received 12 April 2012; revised 18 May 2012; accepted 29 May 2012

\begin{abstract}
Proteus mirabilis, a motile Gram-negative bacterium, represents a common cause of complicated urinary tract infections. Autotransporters are a family of secreted proteins from Gram-negative bacteria that direct their own secretion across the outer membrane (type $\mathrm{V}$ autotransporter secretion mechanism). Serine protease autotransporters of Enterobacteriaceae (SPATEs) include adhesins, toxins, and proteases that can contribute to the virulence. Plasmid-encoded toxin (Pet) is the predominant protein in culture supernatants of enteroaggregative $E$. coli prototype strain 042 and has been extensively studied. Pet toxin is encoded on the 65-MDa adherence-related plasmid of EAEC 042 strain. In this work, Pet protein was found in the supernatant obtained from Proteus mirabilis RTX339 strain isolated from a psychiatric patient suffering complicated urinary tract infections (UTIs). The nucleotide sequence of pet gene was obtained using primers designed from $E$. coli 042 pet gene reported. The alignment of the sequence showed $100 \%$ identity with the pet gene reported. Is important to note that Proteus mirabilis RTX339 pet gene has chromosomal location. The chromosomal location of the gene was established since no plasmids were harbored by this strain.
\end{abstract}

Keywords: Pet; Proteus mirabilis; SPATEs

\section{INTRODUCTION}

Proteus mirabilis is a common cause of urinary tract infections in patients with structural abnormalities of the urinary tract [1]. Urinary tract infections caused by this bacterial pathogen are common and often severe, leading

${ }^{*}$ Corresponding author. to acute pyelonephritis, chronic inflammation, periurethral abscesses, renal failure, and bacteremia. About $90 \%$ of $P$. mirabilis urinary tract infections show renal involvement, and this predilection for the upper urinary tract has been confirmed in animal models, which have also shown that this microorganism is able to invade host kidney cells [2]. P. mirabilis exhibits a form of multicellular behavior known as swarming migration. It is believed that its ability to colonize the urinary tract is associated with its swarming motility [3]. In addition these bacteria colonize the catheter, forming a biofilm [4]. Proteus-associated UTIs may be difficult to treat, and the bacterium persists due to complications associated with this type of infection, including bladder and kidney stone formation (urolithiasis) that can lead to the obstruction of catheters and the urinary tract [5]. Autotransporters are a family of secreted proteins from Gram-negative bacteria that direct their own secretion across the outer membrane (this secretion mechanism has also been described as type $\mathrm{V}$ secretion). The serine protease autotransporters of Enterobacteriaceae (SPATEs) include adhesins, toxins, and proteases that can contribute to the virulence of different E. coli pathotypes [6]. Plasmid-encoded toxin (Pet), first described in enteroaggregative Escherichia coli (EAEC) strain 042 [7], is a $107 \mathrm{KDa}$ secreted protein that damages the human intestinal mucosa by inducing exfoliation of epithelial cells and development of crypt abscesses. The toxin is classified as a SPATE [8]. Pet represented the first enterotoxin within the autotransporter class of secreted proteins. It has also been reported that Pet produces cellular damage associated with fodrin disruption on live HEp-2 cells [9], and in 2002, using an animal model study [10], it was reported histopathological evidence that confirmed the stimulation of mucus hypersecretion, an increased amount of goblet cells and the presence of bacterial aggregates in the apical surface of 
intestinal epithelial cells as well as edema in the submucosa, when BALB/c mice were inoculated with the 042 EAEC strain.

In 2008 [11], six putative Proteus mirabilis autotransporter (ATs) proteins were identified in the complete genome sequence of an uropathogen Proteus mirabilis strain. Pta is an AT with a subtilisin-like protease motif described later as one of the six AT identified in the whole $P$. mirabilis genome [12]. In this work, Pet protein was found in the culture supernatant of Proteus mirabilis strain RTX339 isolated from a psychiatric patient with UTI, and peptide sequences were obtained and compared with the reported sequence described for EAEC 042. Gene pet of Proteus mirabilis RTX339 strain was PCR amplified and sequenced (GenBank acc. no. JX050262). This sequence was compared with the reported sequence for EAEC 042 pet gene.

\section{MATERIALS AND METHODS}

\subsection{Bacterial Strains and Culture Conditions}

Proteus mirabilis strains were isolated from urine of hospitalized infected patients from a psychiatric hospital and from a second level care clinic from Mexico City. Wild-type EAEC strain 042 was used as a Pet producing control strain. Twenty four urine $P$. mirabilis clinical isolates from our collection were additionally screened. Unless otherwise indicated, strains were cultured in MacConkey agar or Luria broth (LB) at $37^{\circ} \mathrm{C}$ and maintained at $-70^{\circ} \mathrm{C}$ in LB-glycerol (1:1).

\subsection{Protein Purification}

Pet protein was obtained from culture supernatants of EAEC 042 and $P$. mirabilis RTX339 strains, precipitated with $75 \%$ ammonium sulfate, and further precipitated with 1.15 and $1.75 \mathrm{M}$ potassium phosphate buffer. The purified proteins were analyzed by sodium dodecyl sulfate (SDS)-6\% polyacrylamide gel electrophoresis (PAGE) [13].

\subsection{Western Immunoblot}

The protein bands obtained by SDS-6\% PAGE were transferred to a PVDF membrane [14]. Membranes were incubated overnight with rabbit antibodies against Pet EAEC 042 protein (diluted 1:500) and visualized with goat anti-rabbit antibodies conjugated with alkaline phosphatase (Kirkegaard \& Perry Laboratories, Gaithersburg, Md.).

\subsection{Protein Sequencing}

The protein bands were carefully excised from Coomassie stained PVDF membranes, distained, reduced, carbami- domethylated, washed, digested with trypsin and extracted according to published procedures [15]. The volume of the extracts was reduced by evaporation in a vacuum centrifuge at ambient temperature and then adjusted to $20 \mu \mathrm{L}$ with $0.1 \%$ formic acid.

LC/MS/MS analysis of triptic peptides were carried out using a NanoAcquity ultraperformance liquid chromatograph (UPLC) (Waters Corporation) coupled to a Q-ToF Synapt High Definition Mass Spectrometer (Waters Corporation) equipped with a NanoLockSpray ion source. The binary solvent system used was $2 \%$ acetonitrile in Milli Q water with $0.1 \%$ formic acid (mobile phase A) and $98 \%$ acetonitrile in Milli Q water with $0.1 \%$ formic acid (mobile phase B). Samples were concentrated and desalted online by injection onto a Symmetry $\mathrm{C}_{18}$ UPLC trapping column (5 $\mu \mathrm{m}$ particle size, $180 \mu \mathrm{m}$ id $\times$ $20 \mathrm{~mm}$ ) (Waters Corporation) at a flow rate of $5 \mu \mathrm{L} / \mathrm{min}$ with a $1 \%$ mobile phase $\mathrm{B}$ composition. After $3 \mathrm{~min}$, the trap column was switched in line with the analytical column. Peptides were separated on a BEH, $\mathrm{C}_{18}$ UPLC column $(1.7 \mu \mathrm{m}$ particle size, $75 \mu \mathrm{m}$ id $\times 100 \mathrm{~mm})$ (Waters Corporation) using a linear gradient of $3 \%-40 \%$ B over a $30 \mathrm{~min}$ period, at a flow rate of $0.3 \mu \mathrm{L} / \mathrm{min}$. followed by a 10 min rinse of $95 \%$ mobile phase B. The column was re-equilibrated at initial conditions (3\% mobile phase B) for $25 \mathrm{~min}$. The mass spectrometer was operated in ESI positive V-mode at a resolution of 10000 full with at half height (fwhh). Spectra were acquired in automated mode using data-dependent acquisition (DDA). [Glu ${ }^{1}$ ] fibrinopeptide B solution (100 fmol/ $\mu \mathrm{L}$ ) was infused through the reference sprayer of the NanoLockSpray source at a flow rate of $0.5 \mu \mathrm{L} / \mathrm{min}$ and was sampled at $30 \mathrm{~s}$ intervals during the acquisition. MS survey scans of $1 \mathrm{~s}$ over the $\mathrm{m} / \mathrm{z}$ range 300 to 1600 were used for peptide detection followed by two MS/MS scans of $1 \mathrm{~s}$ each $(\mathrm{m} / \mathrm{z}$ 50 to 2000) of detected precursors. Collision energies were automatically adjusted based upon the ion charge state and the mass. The five most intensive precursor ions of charge $2^{+}, 3^{+}$or $4^{+}$were interrogated per MS/MS switching event. Dynamic exclusion for $60 \mathrm{~s}$ was used to minimize multiple MS/MS events for the same precursor. The data were postadquisition lock mass corrected using the doubly protonated monoisotopic ion of $\left[\mathrm{Glu}^{1}\right]$ fibrinopeptide [16].

Data Processing and Protein Identification. DDA raw data files were processed and searched using the ProteinLynx Global Server version 2.2 (PLGS) software (Waters Corporation) .The default parameter settings included: Perform lockspray calibration with a lockmass tolerance of 0.1 Da. Background substract type, adaptative. Deisotoping type, medium. Trypsin was set as the digest reagent and one missed cleavage site was allowed. Mass tolerances of $30 \mathrm{ppm}$ and $0.05 \mathrm{Da}$ were used for precursor and product ions, respectively. Carbamidomethyl-cy- 
steine was the fixed modification. Met oxidation and GlnAsn deamidation were set as variable modifications. PLGS was configured to automatically output pkl files which were subsequently database searched by MASCOT search algorithm (Version 1.6b9, Matrix Science, London, UK) available at http://www.matrixscience.com). Mascot searches were conducted using the Proteobacteria (purple bacteria) subset (2745025 sequences, Nov 2009 for $P$. mirabilis RTX334 strain and April 2012 for P. mirabilis RTX339 strain) of the National Center for Biotechnology Information non-redundant database (NCBInr,

http://www.ncbi.nih.gov). Peptide matches with Mascot scores exceeding the $95 \%$ level of confidence were accepted as correct matches. Ion score is $-10^{*} \log (\mathrm{P})$ were $\mathrm{P}$ is the probability that the observed match is a random event. The threshold score in the above conditions were 56 and 57 for $\mathrm{P}<0.05$.

\subsection{Identification System (Vitek)}

Identification of the $P$. mirabilis RTX334 and RTX339 strains was performed by the automated system Vitek. The test cards were inoculated with organisms which were cultured from the nutrient agar tryptic soy agar plates. Organisms were no more than $24 \mathrm{~h}$ old at the time of inoculation. In order to inoculate both cards from the same inoculum, a 1.0 McFarland suspension of each organism was prepared in a sufficient volume of $0.45 \%$ sterile saline to inoculate GNI1 card. The saline was then divided into separate tubes for the inoculation of the individual cards. The use of the transfer tubes, filling module, sealing module, and loading of the GNI1 cards into the reader/incubator tray were performed according to the Vitek operator's manual bio-Merieux Vitek software version 5.01 was used [17].

\subsection{Chromosomal DNA Extraction and PCR}

Chromosomal DNA from $P$. mirabilis strain was extracted using the Wizard Genomic DNA Purification Kit (Promega, Cat. A1120, Promega Corporation, Madison WI, USA), following the instructions of the supplier. PCR was carried out using a PCR QIAGEN Kit (QIAGEN Inc., Valencia CA). The PCR mixture was composed of $1 \times$ PCR buffer, $1 \times \mathrm{Q}$ solution, $0.5 \mu \mathrm{M}$ of each primer (Table 1), $200 \mu \mathrm{M}$ each of the four deoxynucleoside triphosphates and $2.5 \mathrm{U}$ of Taq polymerase. The total volume of each reaction mixture was $25 \mu \mathrm{l}$, employing $1 \mu \mathrm{l}$ of DNA extract as the template. Using a T Personal Thermocycler (Biometra) the thermal cycle program consisted of an initial cycle of $95^{\circ} \mathrm{C}$ for 4 min for denaturation and polymerase activation, 30 cycles of: $95.0^{\circ} \mathrm{C}, 60 \mathrm{~s} ; 58.0^{\circ} \mathrm{C}$, $60 \mathrm{~s} ; 72.0^{\circ} \mathrm{C}, 120 \mathrm{~s}$, and a final extension step of $5 \mathrm{~min}$ at $72.0^{\circ} \mathrm{C}$. Amplified products were subjected to $1.5 \%$ agarose gel electrophoresis. Amplified DNA products of specific size (Table 1) were visualized by UV fluorescence after being stained with ethidium bromide. PCR products were purified using a QIAGEN QIAquick PCR Purification Kit (QIAGEN Inc., Valencia CA), and sequenced in an automatic sequencer ABI PRISM Model 3100 Version 3.7, for pet gene confirmation.

\subsection{Plasmid DNA Extraction}

For plasmid DNA extraction a Plasmid Mini Kit (QIAGEN Inc., Valencia CA.) was used following the manufacture instructions.

\subsection{Anti-Pet Antibodies of P. mirabilis RTX 339 Strain}

P. mirabilis RTX339 Pet protein was injected subcutaneously (using polyacrylamide as adjuvant, the band of Pet protein was cut out and macerated for use) to New Zealand White rabbit weighing between 2.5 and $3.0 \mathrm{~kg}$. Rabbit received subcutaneous boosters of $200 \mathrm{mg}$ of total protein on days $0,15,20$, and 25 . Rabbits were exsanguinated on day 30, and the serum collected was stored at $-70^{\circ} \mathrm{C}$ until use. The Pet protein from EAEC 042 obtained by SDS-6\% PAGE were transferred to a PVDF membrane [14]. Membranes were incubated overnight with rabbit antibodies against Pet RTX339 protein (diluted 1:500) and visualized with goat anti-rabbit antibodies conjugated with alkaline phosphatase (Kirkegaard \& Perry Laboratories, Gaithersburg, Md.).

\section{RESULTS}

\subsection{Electrophoresis and Western Immunoblot}

SDS-PAGE (6\%) electrophoresis showed the protein bands obtained from $P$. mirabilis RTX339 strain supernatant. Different protein bands were observed with different molecular weights; however, a band of special interest with an approximate molecular weight of $108 \mathrm{kDa}$ was

Table 1. Primers used in this work.

\begin{tabular}{ccc}
\hline Primer & Sequence 5'-3' & $\begin{array}{c}\text { Product Size } \\
\text { (bp) }\end{array}$ \\
\hline $\begin{array}{l}\text { Pm787 forward } \\
\text { Pm787 reverse }\end{array}$ & ATCAGCCCCCTTCATTTTATTCTAC & 787 \\
Pm800A forward & AATAAAGGGGTGTTTCAACGA & 800 \\
Pm800A reverse & CTCGATATCTTGAGTTGTATT & \\
Pm800F forward & CTTAATGTCATACAGGCACAG & 800 \\
Pm800R reverse & GGTGAATCCGGCACCGGTAAT & \\
Pm650F forward & GATGGCTCCATCAGCATAGGG & 650 \\
Pm650R reverse & CAGCAGGTTGTTTGAACCTTC & \\
U250F forward & TGACTCTGCATGGATTGAGC & 250 \\
U250R reverse & GACGCATCACTCAGTACAGT & \\
U250F forward & TGACTCTGCATGGATTGAGC & 410 \\
Pm410 reverse & GAAGGAGTAACGGAAGTTGGC & \\
\hline
\end{tabular}


selected for sequencing (Figure 1). The western immunoblot assay showed an antigenic reaction between the protein band $(108 \mathrm{kDa})$ obtained from the supernatants and the anti-pet antibody used (Figure 2).

\subsection{Protein Sequencing}

Mass spectrometry assays were performed to identify the $108 \mathrm{kDa}$ band in both cases (P. mirabilis RTX334 and $P$.

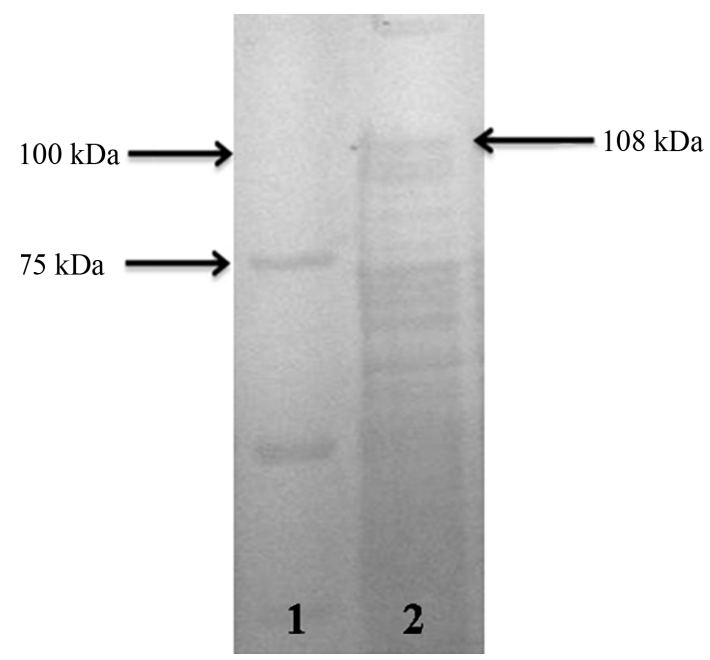

Figure 1. SDS-PAGE (6\%) electrophoresis. Lane 1. Molecular weight marker (Precision plus protein standard, Bio-Rad). Lane 2. P. mirabilis RTX339 strain supernatant protein bands. A protein band with a molecular weight of approximately $108 \mathrm{kDa}$ was observed in the gel. mirabilis RTX339 strains). The peptide sequences obtained identified a protein that has identical sequences when compared with those reported for Pet protein of $E$. coli 042 strain (acc. no. AF056581) [8]. Other proteins sequences were also identified and the results showed that they were reported for Proteus mirabilis HI4320 (Tables 2 and 3).

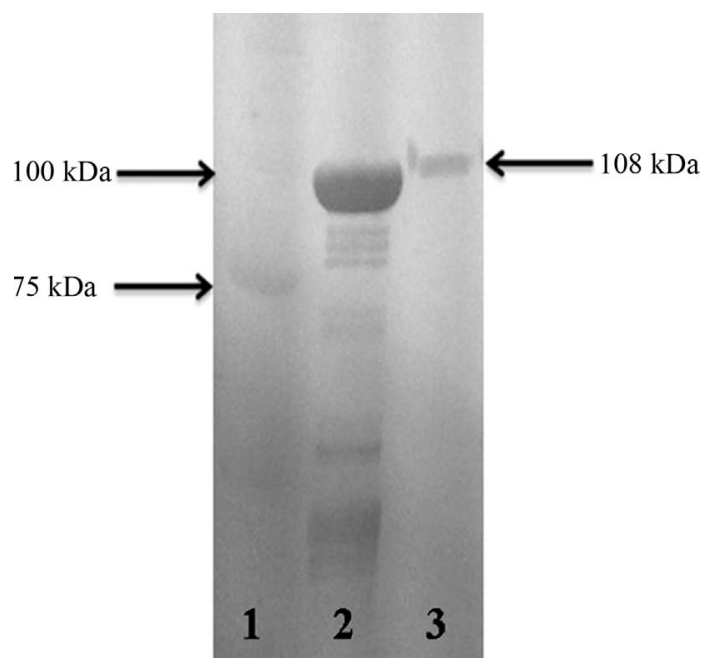

Figure 2. Western immunoblot assay. Lane 1. Molecular weight marker (Precision plus protein standard, Bio-Rad). Lane 2. Pet protein from EAEC 042 used as positive control. Lane 3. Protein band (108 kDa) obtained from the supernatant of Proteus mirabilis RTX339 strain recognized by the antibodies used. Both proteins were recognized by the antibodies raised against the Pet protein of EAEC 042 strain.

Table 2. Identified peptide sequence with mass spectrometry for Pet protein from P. mirabilis RTX334 strain. In this case, individual ions scores $>56$ indicate identity or extensive homology.

\begin{tabular}{lcc}
\hline Acc. no. & Identified protein & Score \\
\hline gi|73921731 & Full=Plasmid-encoded toxin pet & 2702 \\
gi|197284333 & Leucyl-tRNA synthetase [Proteus mirabilis HI4320] & 1227 \\
gi|197285591 & DNA gyrase subunit A [Proteus mirabilis HI4320] & 413 \\
gi|197283930 & Isoleucyl-tRNA synthetase [Proteus mirabilis HI4320] \\
gi|197285894 & Dihydrolipoyllysine-residue acetyltransferase component of pyruvate dehydrogenase complex [Proteus mirabilis HI4320] & 313 \\
gi|197284197 & Glycosyl hydrolase [Proteus mirabilis HI4320] \\
gi|197284466 & 2-oxoglutarate dehydrogenase E1 component [Proteus mirabilis HI4320] & 148 \\
gi|17973460 & Inner membrane RND family protein AcrB [Proteus mirabilis] \\
gi|197285345 & aldehyde-alcohol dehydrogenase [includes: alcohol dehydrogenase, Acetaldehyde dehydrogenase (acetylating), \\
\hline
\end{tabular}


Table 3. Identified peptide sequence with mass spectrometry for Pet protein from P. mirabilis RTX339 strain. In this case, individual ions scores $>57$ indicate identity or extensive homology.

\begin{tabular}{ccc}
\hline Acc. no. & Identified protein & Score \\
\hline gi|73921731 & Full=Plasmid-encoded toxin pet & 2846 \\
gi|197286393 & Aspartate Ammonia Lyase [Proteus mirabilis HI4320] & 192 \\
gi|226329632 & Hypothetical protein PROPEN_03542 [Proteus penneri ATCC 35198] & 106 \\
gi|326794310 & 4-Hidroxythreonine-4-phosphate dehydrogenase [Marinomonas Mediterranea] & 82 \\
gi|197283930 & Isoleucyl t-RNA synthetase [Proteus mirabilis HI4320] & 78 \\
gi|51245430 & Hypothetical protein DP1578 [Desulfotalea psychrophila LSv54] & 72 \\
gi|153873585 & Hypothetical protein BGP_4189 [Beggiatoa sp. PS] & 71 \\
gi|374371354 & Hypothetical protein OR16_38192 [Cupriavidus basilensis OR16] & 67 \\
gi|114563057 & Smr protein/MutS2 [Shewanella frigidimarina NCIMB 400] & 67 \\
\hline
\end{tabular}

\subsection{Vitek Identification}

In order to know whether the strains used were not contaminated, identification with the Vitek automated system was performed. The results showed that the strains were Proteus mirabilis with a $100 \%$ identification.

\subsection{Anti-Pet Antibodies of P. mirabilis RTX 339 Strain}

When anti-pet antibodies obtained from $P$. mirabilis RTX 339 strain were used for the western blot assay, Pet protein of EAEC 042 showed an antigenic reaction with these antibodies as it does with those obtained from EAEC 042. (Figure 3).

\subsection{PCR}

Chromosomal DNA was extracted in order to search for pet gene. This $P$. mirabilis strain does not have plasmids, since no plasmidic DNA was obtained. PCR was performed with the chromosomal DNA, and four set of primers (Table 1) were designed using the peptide sequences obtained from the mass spectrometry results. Five amplicons were obtained, purified and sequenced (Figure 4). When submitted to BLAST analysis results showed a $100 \%$ identity with sequences reported for the pet gene of E. coli 042 (acc. no. AF056581). To determine the prevalence of the pet gene among clinical isolates, PCR assays were performed using a specific primer to amplify a product of 650 bp for pet gene. From our collection, 24 $P$. mirabilis strains isolated from hospitalized patients suffering from UTIs were PCR analyzed, and one strain JTX17 also carried the pet gene within its chromosomal DNA.

\subsection{Nucleotide Sequence Accession Number}

The sequence of the Proteus mirabilis pet gene has been submitted to GenBank under accession no. JX050262.

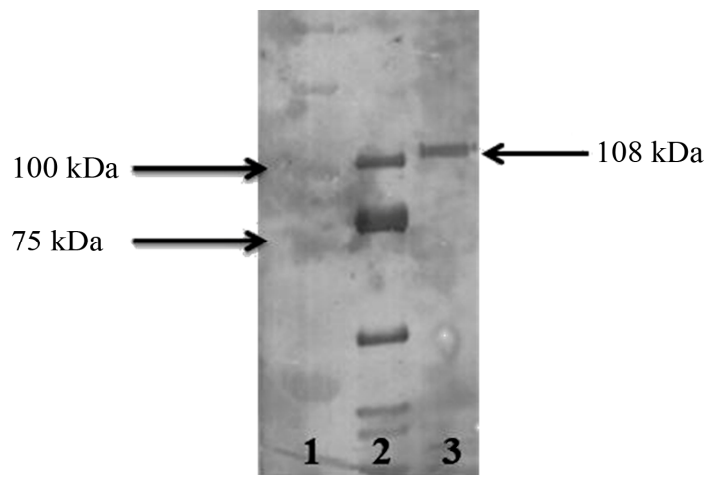

Figure 3. Western immunoblot assay. Lane 1. Molecular weight marker (Precision plus protein standard, BioRad). Lane 2. Pet protein from EAEC 042. Lane 3. Protein band (108 $\mathrm{kDa})$ obtained from the supernatant of Proteus mirabilis RTX339 strain and used as positive control. Both proteins were recognized by antibodies raised against the Pet protein of Proteus mirabilis RTX 339 strain.

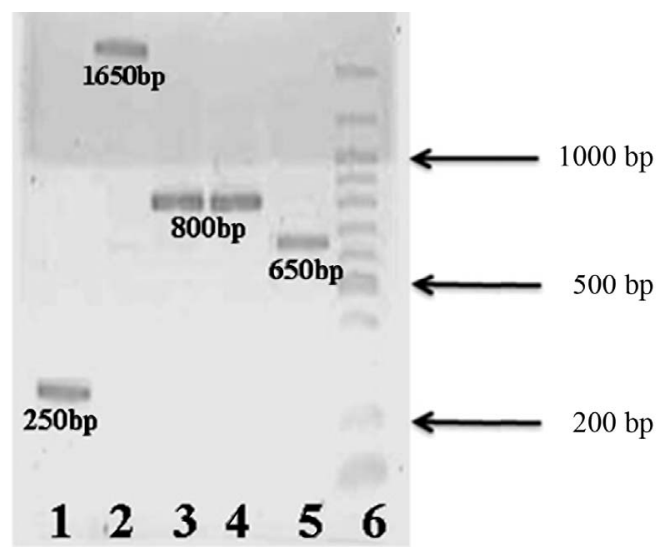

Figure 4. Agarose gel electrophoresis (1.5\%) of PCR products: Lane 1: $250 \mathrm{bp}$ amplified product. Lane 2: 1650 bp amplified product using Pm650F and PM410R primers. Lane 3: 800 bp amplified product using Pm800A primers. Lane 4: 800 bp amplified product using Pm800 primers. Lane 5: 650 bp amplified product. Lane 6: Molecular weight marker (100 bp DNA Ladder, New England BioLabs). 


\section{DISCUSSION}

Proteus mirabilis, a motile Gram-negative bacterium, represents a common cause of complicated urinary tract infections. These infections typically occur in patients with functional or anatomical abnormalities of the urinary tract or patients subjected to long-term catheterizetion [18]. Several virulence factors have been identified and characterized for $P$. mirabilis. These factors include a potent urease that catalyzes formation of ammonia from urea and leads to urinary stone formation, a poreforming hemolysin, ZapA metalloprotease which cleaves both immunoglobulin G (IgG) and IgA, a capsular polysaccharide, four distinct fimbrial types, and peritrichous flagella for swimming and swarming motility [19]. However, Escherichia coli is by far the most common cause of urinary tract infection (UTI), particularly in uncomplicated cases. Strains causing these infections possess traits that distinguish them from commensal strains of $E$. coli and other pathogenic strains such as those causing diarrhea and meningitis. Characteristically, uropathogenic strains of $E$. coli are composed of a restricted number of $\mathrm{O}$ serogroups, produce hemolysin, $\mathrm{P}$ fimbriae, and aerobactin, exhibit serum resistance, and are encapsulated [20]. The plasmid-encoded toxin (Pet) is the predominant protein in culture supernatants of enteroaggregative E. coli prototype strain 042 and has been extensively studied [21]. Pet toxin is encoded on the 65-MDa adherence-related plasmid of EAEC strain 042 [7], however, we found this Pet protein in the supernatant from Proteus mirabilis RTX339 strain isolated from patients with UTIs. Furthermore, we obtained antibodies against Pet protein of P.mirabilis RTX 339 strain and are capable of react and recognize the Pet protein of Escherichia coli 042 type strain, which means that the proteins of both strains share common epitopes, besides they might have a high level of homology, as has been described that some molecules in the case of Escherichia coli have a very similar structure [22]. We also identified the presence of the pet gene in the chromosomal DNA of $P$. mirabilis RTX334, RTX339 and JTX17 strains. The autotransporters are a growing family of proteins, members of which have been identified across the breadth of the gram-negative evolutionary tree; as expected, the phylogeny of the proteins is complex. Evidence for divergent evolution among the autotransporters is provided by examination of the serine protease autotransporters of the Enterobacteriaceae (SPATEs) [23]. This class of proteins, called SPATEs [24], includes the putative virulence factors SepA, SigA, and Pic of Shigella flexneri $[22,25,26]$, Sat of uropathogenic E. coli [27], EspP of Shiga-toxin producing E. coli [28], EspC of enteropathogenic E. coli $[29,30]$, and Pet and Pic of EAEC [7,22,31]. Pearson et al., identified six putative Proteus mirabilis autotransporter proteins in the com- plete genome sequence of an uropathogen Proteus mirabilis HI4320 strain [11]. Pta is an autotransporter protein also found in P. mirabilis strains HI4320 that has a cytotoxin and agglutinin properties when tested in vitro using human bladder and kidney epithelial cell lines, confirming that autotransporter proteins could be cytotoxins [12]. Since no plasmidic DNA was obtained from these two strains the gene is located within the chromosomal DNA and this represents a difference when compared with the reported for pet gene from E. coli 042 because it is located within the reported plasmid sequence [7]. Due that Pet toxin degrades fodrin protein that is part of the cytoskeleton of the intestinal epithelial cells as reported by Villaseca, et al. [9], we suggest that this type of toxin could also caused alterations in the urinary epithelial cells as well. Pet protein has great homology with Sat an autotransporter toxin which is also part of the SPATE's family and was also found in the chromosome of uropathogenic E. coli, causing alterations to the urinary tract epithelium during urinary infections [22]. However, despite the complexity of autotransporters, several inferences can be made from the phylogenetic study of these proteins. The influence of horizontal gene transfer on autotransporter distribution within the gram-negative bacteria is illustrated by the immunoglobulin A1 (IgA1) proteases; despite the fact that Neisseria and Haemophilus are located on distinct branches of the evolutionary tree, members of the IgA1 proteases can be found in both species. Members of this closely related group of proteins have evolved specific and distinct functions which are adaptive for the particular niche occupied by the pathogen even though they possess significant homology ( $40 \%$ to $100 \%$ identity) through the complete protein [21]. Further studies are ongoing in order to investigate if this chromosomal protein is located within a pathogenicity island with transposable elements or in phages.

Is the first time that Pet toxin has been described in Proteus mirabilis strains. The gene that encodes the Pet protein has a chromosomal location.

\section{ACKNOWLEDGEMENTS}

L. R. Gutiérrez-Lucas is a Ph.D. student at the Doctorado en Ciencias Biológicas of Universidad Autónoma Metropolitana, Unidad Xochimilco, and was supported by grant 171724 of CONACYT.

\section{REFERENCES}

[1] Chippendale, G.R., Warren, J.W., Trifillis, A.L. and Mobley, H.L.T. (1994) Internalization of Proteus mirabilis by human renal epithelial cells. Infection and Immunity, 62, 3115-3121.

[2] Allison, C., Coleman, N., Jones, P.L. and Hughes, C. 
(1992) Ability of Proteus mirabilis to invade human urothelial cells is coupled to motility and swarming differentiation. Infection and Immunity, 60, 4740-4746.

[3] Jiang, S., Lin, T., Wang, W., Liu, M., Hsueh, P. and Liaw, S. (2010) Characterization of UDP-Glucose dehydrogenase and UDP-Glucose pyrophosphorylase mutants of Proteus mirabilis: Defectiveness in polymyxin B resistance, swarming, and virulence. Antimicrobial Agents and Chemotherapy, 54, 2000-2009.

doi:10.1128/AAC.01384-09

[4] Stickler, D.J., Jones, S.M., Adusei, G.O. and Waters, M.G. (2006) A sensor to detect the early stages in the development of crystalline Proteus mirabilis biofilm on indwelling bladder catheters. Journal of Clinical Microbiology, 38, 1540-1542.

doi:10.1128/JCM.44.4.1540-1542.2006

[5] Jacobsen, S.M., Stickler, D.J., Mobley, H.L.T. and Shirtliff, M.E. (2008) Complicated catheter-associated urinary tract infections due to Escherichia coli and Proteus mirabilis. Clinical Microbiology Reviews, 21, 26-59. doi:10.1128/CMR.00019-07

[6] Restieri. C., Garriss, G., Locas, M. and Dozois, C. (2007) Autotransporter-encoding sequences are phylogenetically distributed among Escherichia coli clinical isolates and reference strains. Applied and Environmental Microbiology, 73, 1553-1562. doi:10.1128/AEM.01542-06

[7] Eslava, C., Navarro-García, F., Czeczulin, J., Henderson, I., Cravioto, A., et al. (1998) Pet, an autotransporter enterotoxin from enteroaggregative Escherichia coli. Infection and Immunity, 66, 3155-3163.

[8] Navarro-García, F., Canizales-Roman, A., Burlingame, K., Teter, K. and Vidal, J. (2007) Pet, a non-AB toxin, is transported and translocated into epithelial cells by a retrograde trafficking pathway. Infection and Immunity, 75, 2101-2109. doi:10.1128/IAI.01515-06

[9] Villaseca, J., Navarro-García, F., Mendoza-Hernández, G., Nataro, J., Cravioto, A., et al. (2000) Pet toxin from enteroaggregative Escherichia coli produces cellular damage associated with fodrin disruption. Infection and Immunity, 68, 5920-5927. doi:10.1128/IAI.68.10.5920-5927.2000

[10] Sainz, T., Perez, J., Fresan, Ma.C., Flores, V., Jimenez, L., et al. (2002) Histological alterations and immune response induced by Pet toxin during colonization with Enteroaggregative Escherichia coli (EAEC) in a mouse model infection. Korean Journal of Microbiology, 40, 9197.

[11] Pearson, M., Sebaihia, M., Churcher, C., Quail, M., Seshasayee, A., et al. (2008) Complete genome sequence of uropathogenic Proteus mirabilis, a master of both adherence and motility. Journal of Bacteriology, 190, 40274037. doi:10.1128/JB.01981-07

[12] Alamuri, P. and Mobley, H.L.T. (2008) A novel autotransporter of uropathogenic Proteus mirabilis is both a cytotoxin and an agglutinin. Molecular Microbiology, 68, 997-1017. doi:10.1111/j.1365-2958.2008.06199.x

[13] Laemmli, U.K. (1970) Cleavage of structural proteins during the assembly of the head of bacteriophage T4. Nature, 227, 680-685. doi:10.1038/227680a0
[14] Towbin, H., Staehelin, T. and Gordon, J. (1979) Electrophoretic transfer of proteins from polyacrylamide gels to nitrocellulose sheets: Procedure and some applications. Proceedings of the National Academy of Sciences USA, 76, 4350-4354. doi:10.1073/pnas.76.9.4350

[15] Bienvenut, W.V., Sanchez, J.C., Karmime, A., Rougue, V., Rose, K., et al. (1999) Toward a clinical molecular scanner for proteome research: Parallel protein chemical processing before and during western blot. Analytical Chemistry, 21, 4800. doi:10.1021/ac990448m

[16] Kinter, M., Sherman, N.E., Desiderio, D.M. and Nibbering, N.M.M. (2000) In protein sequencing and identification using tandem mass spectrometry. John Wiley-Interscience, Inc., New York, 147-165.

[17] Bourbeau, P. and Heiter, B. (1998) Comparison of Vitek GNI and GNI+ Cards for Identification of Gram-Negative Bacteria. Journal of Clinical Microbiology, 36, 27752777.

[18] Nielubowicz, G., Smith, S. and Mobley, H.L.T. (2010) Zinc uptake contributes to motility and provides a competitive advantage to Proteus mirabilis during experimental urinary tract infection. Infection and Immunity, 78, 2823-2833. doi:10.1128/IAI.01220-09

[19] Jansen, A., Lockatell, C., Johnson, D. and Mobley, H.L.T. (2003) Visualization of Proteus mirabilis morphotypes in the urinary tract: The elongated swarmer cell is rarely observed in ascending urinary tract infection. Infection and Immunity, 71, 3607-3613. doi:10.1128/IAI.71.6.3607-3613.2003

[20] Guyer, D., Kao, J. and Mobley, H.L.T. (1998) Genomic analysis of a pathogenicity island in uropathogenic Escherichia coli CFT073: Distribution of homologous sequences among isolates from patients with pyelonephritis, cystitis, and catheter-associated bacteriuria and from fecal samples. Infection and Immunity, 66, 4411-4417.

[21] Dutta, P., Sui, B. and Nataro, J. (2003) Structure-function analysis of the enteroaggregative Escherichia coli plasmid-encoded toxin autotransporter using scanning linker mutagenesis. Journal of Biological Chemistry, 278, 39912-39920. doi:10.1074/jbc.M303595200

[22] Henderson, I. R., Czeczulin, J., Eslava, C., Noriega, F. and Nataro, J. (1999) Characterization of Pic, a secreted protease of Shigella flexneri and enteroaggregative Escherichia coli. Infection and Immunity, 67, 5587-5596.

[23] Henderson, I. and Nataro, J. (2001) Virulence functions of autotransporter proteins. Infection and Immunity, 69, 1231-1243. doi:10.1128/IAI.69.3.1231-1243.2001

[24] Sui, B., Dutta, P. and Nataro, J. (2003) Intracellular expression of the plasmid-encoded toxin from enteroaggregative Escherichia coli. Infection and Immunity, 71, 5364-5370. doi:10.1128/IAI.71.9.5364-5370.2003

[25] Al-Hasani, K., Henderson, I., Sakellaris, H., Rajakumar, K., Grant, T., et al. (2000) The sigA gene which is borne on the she pathogenicity island of Shigella flexneri 2a encodes an exported cytopathic protease involved in intestinal fluid accumulation. Infection and Immunity, $\mathbf{6 8}$ 2457-2463. doi:10.1128/IAI.68.5.2457-2463.2000

[26] Benjelloun-Touimi, Z., Sansonetti, P. and Parsot, C. 
(1995) SepA, the major extracellular protein of Shigella flexneri: autonomous secretion and involvement in tissue invasion. Molecular Microbiology, 17, 123-135. doi:10.1111/j.1365-2958.1995.mmi_17010123.x

[27] Guyer, D.M., Henderson, I.R., Nataro, J.P., Mobley and H.L. (2000) Identification of sat, an autotransporter toxin produced by uropathogenic Escherichia coli. Molecular Microbiology, 38, 53-66. doi:10.1046/j.1365-2958.2000.02110.x

[28] Brunder, W., Schmidt, H. and Karch, H. (1997) EspP, a novel extracellular serine protease of enterohaemorrhagic Escherichia coli O157:H7 cleaves human coagulation factor V. Molecular Microbiology, 24, 767-778. doi:10.1046/j.1365-2958.1997.3871751.x

[29] Mellies, J.L., Navarro-García, F., Okeke, I., Frederickson, J., Nataro, J.P., et al. (2001) EspC pathogenicity island of enteropathogenic Escherichia coli encodes an enterotoxin. Infection and Immunity, 69, 315-324. doi:10.1128/IAI.69.1.315-324.2001

[30] Stein, M., Kenny, B., Stein, M.A. and Finlay, B.B. (1996) Characterization of EspC, a 110-kilodalton protein secreted by enteropathogenic Escherichia coli which is homologous to members of the immunoglobulin A protease-like family of secreted proteins. Journal of Bacteriology, 178, 6546-6554.

[31] Behrens, M., Sheikh, J. and Nataro, J. P. (2002) Re- gulation of the overlapping pic/set locus in Shigella flexneri and enteroaggregative Escherichia coli. Infection and Immunity, 70, 2915-2925. doi:10.1128/IAI.70.6.2915-2925.2002 